\title{
Preface: Studies on Inverse Problems with Applications: a Collection of Papers from Chinese Scholars
}

\author{
Gang BAO ${ }^{1}$, Jin CHENG $^{2,3}$, Ji-jun LIU ${ }^{4}$, Bo ZHANG ${ }^{5}$ \\ ${ }^{1}$ Zhejiang University, Hangzhou 200433, China (E-mail: baog@zju.edu.cn) \\ ${ }^{2}$ School of Mathematical Sciences, Fudan University, Shanghai 200433, China \\ ${ }^{3}$ School of Mathematics, Shanghai University of Finance and Economics, Shanghai 200433, China \\ (E-mail: jcheng@fudan.edu.cn) \\ ${ }^{4}$ School of Mathematics, Southeast University, Nanjing 210096, China (E-mail: jjliu@seu.edu.cn) \\ ${ }^{5}$ Academy of Mathematics and Systems Science, Chinese Academy of Sciences, Beijing 100190, China \\ (E-mail: b.zhang@amt.ac.cn)
}

With the promotion by both mathematics itself and the practical requirements from modern society, the interest of studies on inverse problems and ill-posed problems, at home and abroad, has been flourishing in recent decades. The intrinsic mathematical reasons for the studies on inverse problems come from the fact that most of the inverse problems are ill-posed, i.e., the existence, uniqueness and stability of the solution cannot be ensured due to the configurations of the problems themselves. The characteristic of the ill-posedness for inverse problems makes it hard to construct the (generalized) solutions, especially to keep the solutions stable with respect to the noisy input data. To overcome these difficulties, some regularizing techniques should be introduced, which are closely related to many mathematical branches such as partial differential equations (PDEs), functional analysis, optimizations and numerical analysis.

The other motivations for the studies on inverse problems are important engineering problems arising in geophysics, medical imaging, remote sensing, detection of contamination sources and so on. The common features of these practical problems include the insufficiency (or over-specification) of input data and the instability of the solutions. A typical problem is the detection of the structure under the earth in geophysics, where we need to obtain some information about the physical parameters from noisy incomplete data measured on the surface of the earth. Such parameters can help us to locate the position and amount of the geophysical sources such as petroleum and coal.

Due to these reasons, studies on inverse and ill-posed problems in China develop very quickly, with some fundamental influences domestically and internationally. As an indication of such deep influences, several Chinese scholars have entered the editorial board of international scientific journals related to inverse and ill-posed problems. Also there are already around 200 participants for Chinese Annual Conference on Inverse Problems and Related Topics in recent years.

In this special issue, we are happy to collect 13 papers from Chinese scholars. Roughly speaking, these papers cover three different topics, namely, scattering and inverse scattering problems by different kinds of waves (paper 1-paper 8), sources detection by diffusion processes (paper 9-paper 11), and imaging techniques for bio-tissues (paper 12-paper 13). All these works are of clear engineering backgrounds and require mathematical methods related to PDEs and regularization as well as numerical analysis. We expect these works are helpful for further researches for those working on inverse problems, especially for younger scholars.

Finally, we would like to express our deep thanks to Academician Zhi-Ming Ma, the Editor-

Manuscript received on October 27, 2019. Accepted on November 21, 2019. 
in-Chief of Acta Mathematicae Applicatae Sinica (English Series) for the invitation to organize this special issue and to Acta Mathematicae Applicatae Sinica (English Series) for providing the forum for presenting the recent works in inverse problems by Chinese scholars. 